\title{
NRF2 Activation Inhibits Both TGF- $\beta 1$ - and IL-13-Mediated Periostin Expression in Fibroblasts: Benefit of Cinnamaldehyde for Antifibrotic Treatment
}

\author{
Yasutaka Mitamura $(\mathbb{D}$, Mika Murai, Chikage Mitoma, and Masutaka Furue \\ Department of Dermatology, Graduate School of Medical Sciences, Kyushu University, Fukuoka, Japan \\ Correspondence should be addressed to Yasutaka Mitamura; m.yasutaka47@gmail.com \\ Yasutaka Mitamura and Mika Murai contributed equally to this work.
}

Received 18 March 2018; Revised 5 June 2018; Accepted 13 June 2018; Published 7 August 2018

Academic Editor: Javier Egea

Copyright (c) 2018 Yasutaka Mitamura et al. This is an open access article distributed under the Creative Commons Attribution License, which permits unrestricted use, distribution, and reproduction in any medium, provided the original work is properly cited.

Systemic fibrosing or sclerotic disorders are life-threatening, but only very limited treatment modalities are available for them. In recent years, periostin (POSTN), a major extracellular matrix component, was established by several studies as a novel key player in the progression of systemic fibrotic disease. In this research, we revealed the involvement of oxidative stress in the expression of POSTN induced by TGF- $\beta 1$ and IL-13 in dermal fibroblasts. We found that the antioxidant cinnamaldehyde activated the NRF2/HMOX1 pathway. Cinnamaldehyde also alleviated TGF- $\beta 1$ - and IL-13-mediated production of reactive oxygen species and subsequent POSTN upregulation in dermal fibroblasts. In contrast, NRF2 silencing abolished the cinnamaldehyde-mediated downregulation of POSTN. These results suggest that cinnamaldehyde is a broad inhibitor of POSTN expression covering both TGF- $\beta 1$ and IL-13 signaling. Cinnamaldehyde may thus be beneficial for the treatment of systemic fibrotic diseases.

\section{Introduction}

Periostin (POSTN) is a matricellular protein that is profoundly involved in the development and progression of fibrotic diseases as well as allergy and oncogenesis [1-5]. In Postn-deficient mice, bleomycin-induced fibrosis is canceled compared to wild-type control mice [4]. Moreover, the expression of POSTN is upregulated in the lesional skin of scleroderma patients $[4,6,7]$. Serum POSTN levels are also elevated in scleroderma as well as in pulmonary fibrosis $[7,8]$. It has also been shown that the downregulation of POSTN attenuates the profibrotic response of hepatic stellate cells [9].

Fibrosis is a characteristic feature in the pathogenesis of a wide spectrum of diseases, including systemic sclerosis, pulmonary fibrotic disorders, renal fibrotic disease, and liver cirrhosis. Although antifibrotic treatments are currently limited [10-18], recent studies have demonstrated that the activation of nuclear factor erythroid-derived 2-like 2
(NRF2) ameliorates profibrotic processes in the lung, kidney, liver, heart, intestine, and skin [19-27]. NRF2 is a master antioxidant transcription factor that upregulates the transcription of various genes encoding antioxidant enzymes such as HMOX1 and NQO1 $[28,29]$. In parallel with this, the antioxidant phytochemical cinnamaldehyde (CIN) activates NRF2, induces its cytoplasmic-to-nuclear translocation, and upregulates HMOX1 expression in human keratinocytes and human dental pulp cells $[29,30]$. In addition, the CIN-containing traditional herbal Kampo medicine Keishi-bukuryo-gan inhibits collagen production in cultured fibroblasts obtained from scleroderma patients [31]. Moreover, this kind of Kampo medicines is effective at reducing total skin thickness score in some scleroderma patients [32]. However, to the best of our knowledge, no reports have addressed whether NRF2 affects POSTN expression.

The expression of POSTN is upregulated by two distinct cytokine-mediated pathways, namely, transforming growth factor- $\beta 1$ (TGF- $\beta 1$ ) and interleukin-13 (IL-13)/IL-4 
TABLE 1: Primer sequences used for real-time quantitative RT-PCR.

\begin{tabular}{lcc}
\hline Target cDNA & Forward sequence & Reverse sequence $^{\text {Human GAPDH }}$ \\
Human POSTN & $5^{\prime}$-TGCACCACCAACTGCTTAGC-3' & $5^{\prime}$-GGCATGGACTGTGGTCATGAG-3 \\
Human NRF2 & $5^{\prime}$-CAGAGAAATCCCTCCATGAAA-3' & $5^{\prime}$-CAGGAGCTCTTTCAAGTCTGC-3' \\
Human HMOX1 & $5^{\prime}$-CTTGGCCTCATGATTCTGAAGTG-3' & $5^{\prime}$-CCTGAGATGGTGACAAGGGTTGTA-3' \\
Human NQO1 & $5^{\prime}$-AGTCTTCGCCCCTGTCTACT-3 ${ }^{\prime}$ & $5^{\prime}$-GCTGGTGTGTAGGGGATGAC-3' \\
Human TNC & $5^{\prime}$-GGATTGGACCGAGCTGGAA-3' & $5^{\prime}$-AATTGCAGTGAAGATGSSGGCAAC-3' \\
Human VEGFA & $5^{\prime}$-GTCACCGTGTCAACCTGATG-3' & $5^{\prime}$-GCCTGCCTTCAAGATTTCTG-3' \\
Human CTGF & $5^{\prime}$-AGGCCAGCACATAGGAGAGA-3' & $5^{\prime}$-TTTCTTGCGCTTTCGTTTTT-3' \\
\hline
\end{tabular}

$[9,33,34]$. In this study, we demonstrated for the first time that CIN-mediated NRF2 activation inhibited the POSTN expression induced by TGF- $\beta 1$ as well as by IL- 13 signaling. The dual inhibitory actions of CIN-NRF2 activation suggest that CIN-containing medicaments could have therapeutic benefit for treating fibrotic disorders.

\section{Materials and Methods}

2.1. Reagents and Antibodies. Cinnamaldehyde was obtained from Sigma-Aldrich (St. Louis, MO, USA) and dissolved in dimethyl sulfoxide (DMSO; Sigma-Aldrich) or in $100 \%$ ethanol (Wako, Osaka, Japan) at a concentration of $5 \mathrm{mM}$. Anti-NRF2 rabbit polyclonal antibody (H-300) was obtained from Santa Cruz Biotechnology (Dallas, TX, USA), and horseradish peroxidase- (HRP-) linked anti-rabbit IgG antibody (\#7074) was purchased from Cell Signaling Technology (Danvers, MA, USA). For Western blotting analysis, an anti-NRF2 rabbit polyclonal antibody (PA5-27882; Thermo Fisher Scientific), anti-HMOX1 rabbit polyclonal antibody (ab137749; Abcam), anti-NQO1 mouse monoclonal antibody (ab28947; Abcam), and anti-GAPDH (14c10) rabbit monoclonal antibody (\#2118; Cell Signaling Technology) were used.

2.2. Cell Culture. Normal human dermal fibroblasts (NHDFs) were purchased from Lonza (Basel, Switzerland) and were maintained in accordance with the vendor's recommendations. They were pretreated with the indicated concentration of CIN for $30 \mathrm{~min}$ and then treated with $5 \mathrm{ng} / \mathrm{mL}$ TGF- $\beta 1$ or IL-13 (PeproTech, Rocky Hill, NJ, USA) for $48 \mathrm{~h}$. For some experiments, NHDFs were treated with CIN $(25 \mu \mathrm{M})$ for $6 \mathrm{~h}$. Then, RNA extracts were subjected to quantitative reverse transcription PCR (qRT-PCR). For knockdown experiments, NHDFs were cultured with small interfering RNA (siRNA) of NRF2 for $24 \mathrm{~h}$, followed by stimulation with $5 \mathrm{ng} / \mathrm{mL}$ TGF- $\beta 1$ or IL- 13 for $48 \mathrm{~h}$. Then, RNA extracts were subjected to qRT-PCR. For a reconstitution experiment, NHDFs were cultured with siRNA of NRF2 for $24 \mathrm{~h}$, followed by pretreatment with $25 \mu \mathrm{M}$ CIN for $30 \mathrm{~min}$ and then stimulation with $5 \mathrm{ng} / \mathrm{mL}$ TGF- $\beta 1$ or $50 \mathrm{ng} / \mathrm{mL}$ IL-13 for $48 \mathrm{~h}$. RNA extracts and the supernatants were subjected to qRT-PCR or ELISA, respectively.
2.3. Immunofluorescence. NHDFs were placed on an eightwell $\mu$-slide (Ibidi, Munich, Germany) and at subconfluence were treated with $25 \mu \mathrm{M}$ CIN for $3 \mathrm{~h}$. Then, the cells were washed with PBS, fixed with acetone for $10 \mathrm{~min}$, and blocked with 5\% $(w / v)$ bovine serum albumin in PBS for $30 \mathrm{~min}$. Samples were incubated with a primary rabbit anti-NRF2 antibody or control IgG (Abcam, Cambridge, UK). Specific binding was detected using an HRP-conjugated goat antirabbit antibody, followed by tyramide labeling with greenfluorescent Alexa Fluor 488 (Molecular Probes, Eugene, OR, USA), in accordance with the manufacturer's protocol. Samples were covered with UltraCruz ${ }^{\mathrm{TM}}$ mounting medium containing $4^{\prime}$,6-diamidino-2-phenylindole (DAPI) (Santa Cruz Biotechnology) to preserve fluorescence. The fluorescence images were acquired using an EVOS FL cell imaging system (Life Technologies, Carlsbad, CA, USA).

2.4. Detection of Reactive Oxygen Species (ROS) Production. NHDFs were cultured in a 48 -well plate for $24 \mathrm{~h}$. Then, the cells were washed with PBS and stimulated with $20 \mathrm{ng} / \mathrm{mL}$ TGF- $\beta 1$ or $50 \mathrm{ng} / \mathrm{mL}$ IL-13 (PeproTech) for $20 \mathrm{~min}$. Detection of ROS production was performed under a microscope using an Image-IT ${ }^{\mathrm{TM}}$ LIVE Green Reactive Oxygen Species Detection Kit (Thermo Fisher Scientific, Rockford, IL, USA). Briefly, the oxidation-sensitive dye 5-(and-6)-carboxy-20,70-dichlorodihydrofluorescein diacetate (carboxy$\mathrm{H}_{2}$ DCFDA) (Molecular Probes) was used to quantify ROS levels in live cells. Cells were incubated with Dulbecco's phosphate-buffered saline (DPBS; Thermo Fisher Scientific) with calcium chloride and magnesium chloride containing carboxy- $\mathrm{H}_{2} \mathrm{DCFDA}(25 \mathrm{mM})$ for $25 \mathrm{~min}$ at $37^{\circ} \mathrm{C}$. Then, the medium was changed to DPBS containing Hoechst 33342 (Thermo Fisher Scientific) as a blue-fluorescent, cell-permeant nucleic acid stain. The fluorescence images were acquired using an EVOS FL cell imaging system (Life Technologies).

2.5. Knockdown of $m R N A$ by siRNA. siRNA oligonucleotides were purchased from Dharmacon/GE Healthcare (Lafayette, CO, USA). Cells were transfected with $5 \mathrm{nM}$ ON-TARGET plus siRNA for NRF2 or control for $24 \mathrm{~h}$ in the presence of RNAiMAX reagent (Thermo Fisher Scientific), in 


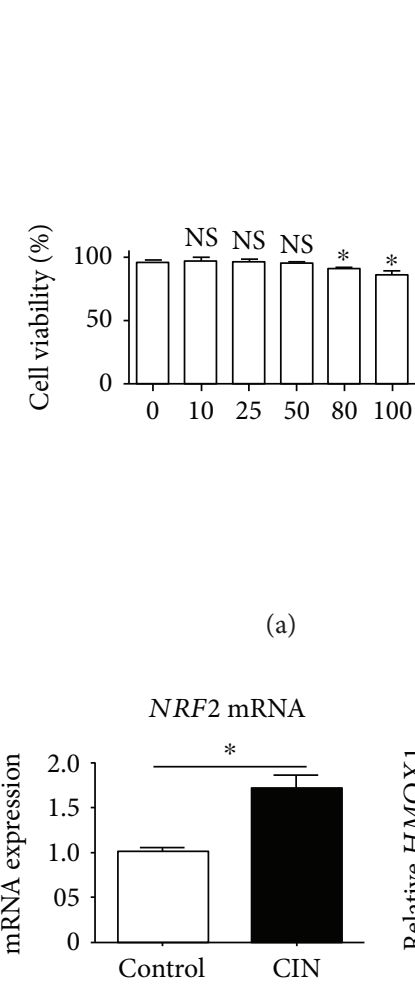

(c)

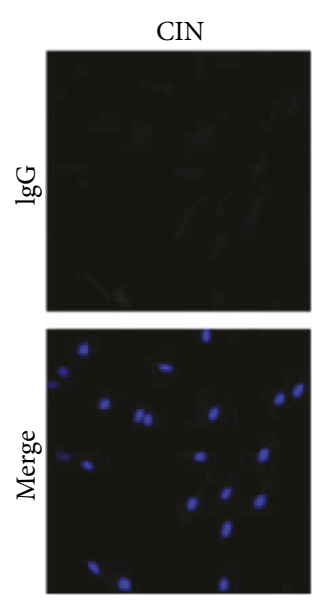

HMOX1 mRNA

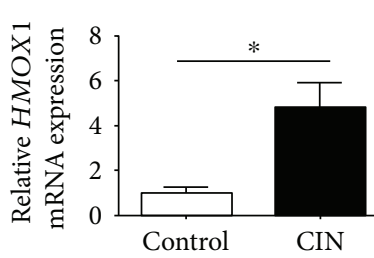

(d)

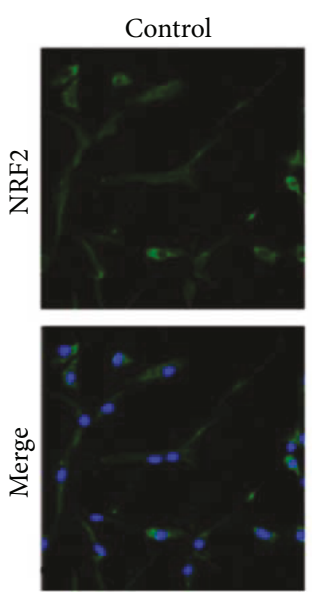

(b)

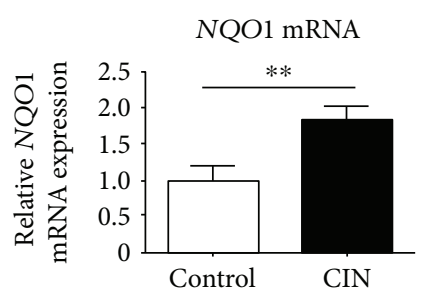

(e)
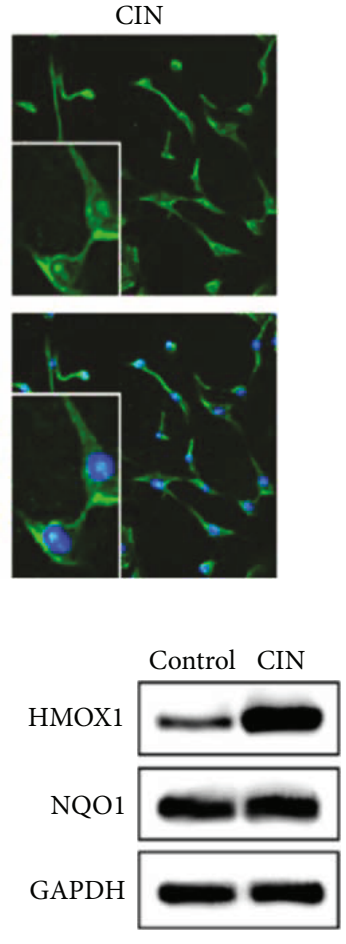

(f)

FIGURE 1: Activation of the NRF2/HMOX1 and NQO-1 pathway by cinnamaldehyde. (a) Effects of cinnamaldehyde (CIN) on the viability of NHDFs. (b) NHDF cells were treated with DMSO (control) or CIN $(25 \mu \mathrm{M})$ for $3 \mathrm{~h}$ and fixed. Cells were then stained with the anti-NRF2 antibody or control IgG (green) and DAPI (blue) and visualized by fluorescence microscopy. (c-e) NHDF cells were treated with DMSO (control) or CIN $(25 \mu \mathrm{M})$ for $6 \mathrm{~h}$, after which total RNA was extracted. (c) NRF2 mRNA expression, (d) HMOX1 mRNA expression, and (e) NQO1 mRNA expression are depicted. (f) NHDF cells were treated with $100 \%$ ethanol (control) or CIN (25 $\mu \mathrm{M})$ for $24 \mathrm{~h}$, after which whole cell lysates were extracted. The expression of HMOX1, NQO1, and GAPDH was depicted. The values were adjusted by GAPDH expression. The same experiments were performed three times. ${ }^{*} P<0.05,{ }^{* *} P<0.01$. NS: not significant.

accordance with the manufacturer's instructions. Silencing of target genes was confirmed by qRT-PCR.

2.6. Quantitative Real-Time Polymerase Chain Reaction ( $q R T-P C R)$. Total RNA was isolated using RNAiso PLUS (Takara Bio, Otsu, Japan) and reverse-transcribed with the RNA-direct ${ }^{\mathrm{TM}}$ Realtime PCR Master Mix (Toyobo, Osaka, Japan). The PCR reactions were performed on a StepOnePlus Real-Time PCR System (Life Technologies) using the THUNDERBIRD SYBR qPCR Mix (Toyobo). Threshold cycles of primer probes were normalized to a housekeeping gene (glyceraldehyde-3-phosphate dehydrogenase (GAPDH)), and relative values were calculated. Primers for qRT-PCR are described in Table 1.

2.7. Enzyme-Linked Immunosorbent Assay (ELISA) for Periostin Production. ELISA for POSTN was performed using two different anti-POSTN Abs, SS18A (the capture antibody) and SS17B (the detection antibody) (Shino-Test, Tokyo, Japan), as previously described [35].

2.8. Western Blotting Analysis. Western blotting was performed as previously described [36]. The antibodies (Abs) used in this study were against NRF2 (1:1000 dilution), HMOX1 (1:1000 dilution), NQO1 (1:1000 dilution), and
GAPDH (1:1000 dilution). The HRP-conjugated antimouse or anti-rabbit IgG antibody (Cell Signaling Technology) was used as a secondary antibody.

2.9. Statistical Analysis. Data are presented as mean \pm SD. Statistical analyses were performed using Prism 5.0 software (GraphPad Software, La Jolla, CA, USA). The significance of differences was assessed using an unpaired one-tailed or two-tailed Student's $t$-test. Values of $P<0.05$ were considered statistically significant.

\section{Results}

3.1. CIN Activates NRF2 Signaling and Induces HMOX1 Production in Dermal Fibroblasts. CIN induces the cytoplasmic-to-nuclear translocation of NRF2 in human keratinocytes and acts against oxidative stress [29]. Here, we first examined the effects of CIN on the viability of cultured fibroblasts. CIN at concentrations up to $50 \mu \mathrm{M}$ did not affect the viability of fibroblasts, as determined by the trypan blue dye exclusion test (Figure 1(a)). As previously demonstrated in keratinocytes [29], CIN also induced the cytoplasmic-to-nuclear translocation of NRF2 in dermal fibroblasts (Figure 1(b)). In addition, CIN induced 

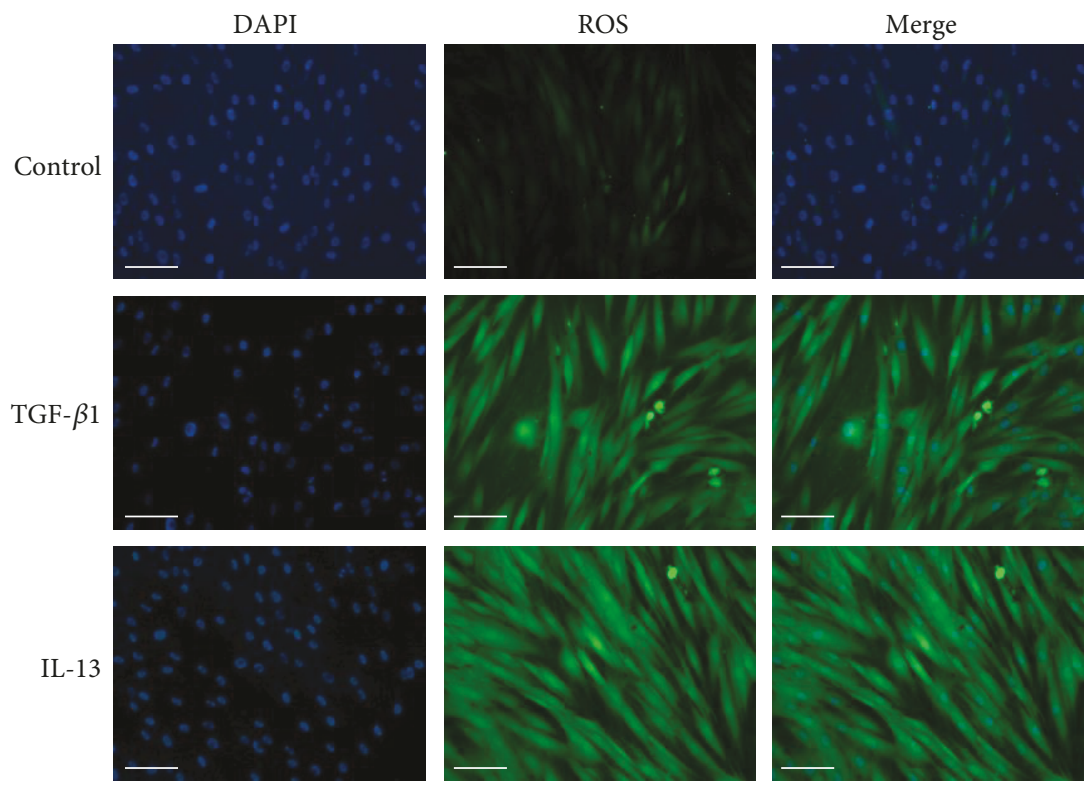

(a)

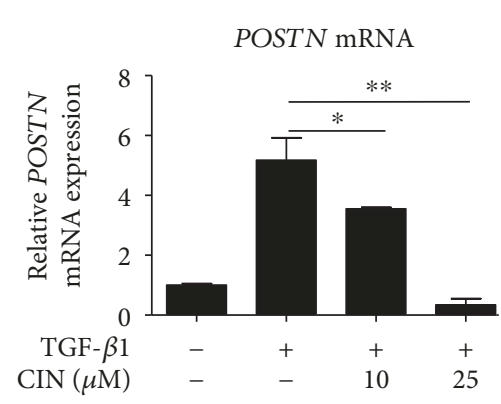

(b)

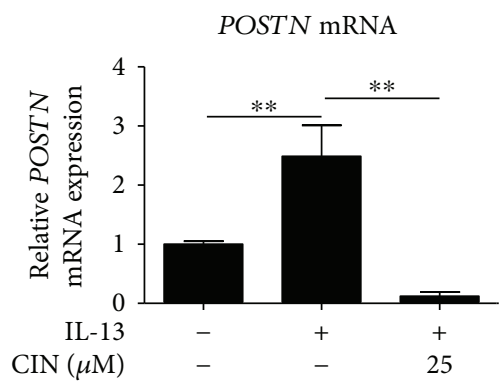

(d)

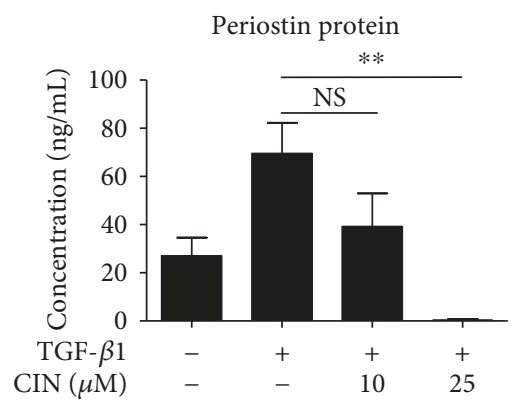

(c)

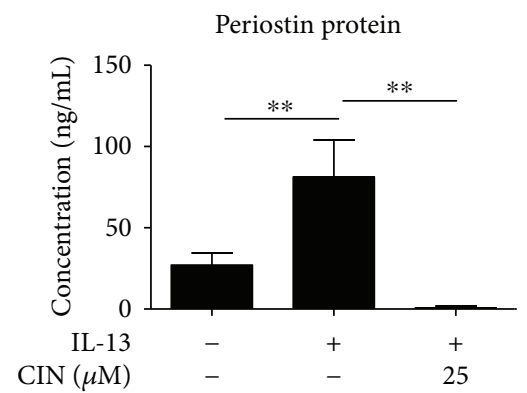

(e)

FIGURE 2: Effects of cinnamaldehyde on TGF- $\beta 1$ - and IL-13-induced expression of periostin mRNA and protein in dermal fibroblasts. (a) NHDFs were stimulated with $20 \mathrm{ng} / \mathrm{mL}$ TGF- $\beta 1$ or $50 \mathrm{ng} / \mathrm{mL}$ IL-13 or unstimulated (control) for 20 min. Cells were incubated with carboxy- $\mathrm{H}_{2}$ DCFDA for $25 \mathrm{~min}$, after which ROS production was visualized by fluorescence microscopy. Scale bars, $200 \mu \mathrm{m}$. (b, c) Effects of cinnamaldehyde (CIN) on TGF- $\beta 1$-induced expression levels of periostin (POSTN) mRNA and protein. (d, e) Effects of CIN on IL-13induced expression of POSTN mRNA and protein. NHDF cells were precultured with the indicated concentration of CIN for 30 min and then treated with $5 \mathrm{ng} / \mathrm{mL}$ TGF- $\beta 1$ or IL-13 or left untreated for $48 \mathrm{~h}$. Expression levels of POSTN mRNA and protein in the cell supernatants are depicted. The values were adjusted by GAPDH expression. The same experiments were performed three times. ${ }^{*} P<0.05$, ${ }^{* *} P<0.01$. NS: not significant.

upregulation of the mRNA expression of NRF2 (Figure 1(c)). In accordance with NRF2 activation, CIN upregulated HMOX1 and NQO1 expression at the mRNA level, but only HMOX1 expression at the protein level (Figures 1(d)-1(f)). These results demonstrate that CIN activates the primarily NRF2/HMOX1 antioxidant pathway in fibroblasts.
3.2. CIN Inhibits POSTN Expression Induced by TGF- $\beta 1$ and IL-13 in Dermal Fibroblasts. In previous studies, it was demonstrated that oxidative stress induces the expression of POSTN in myocardial fibroblasts and human umbilical vein endothelial cells $[37,38]$. Therefore, to further evaluate the antioxidant activities of CIN, we investigated whether CIN 


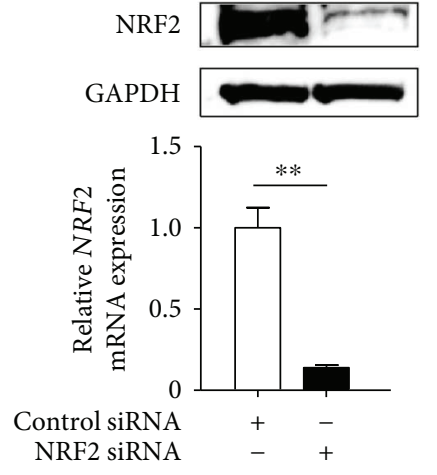

(a)

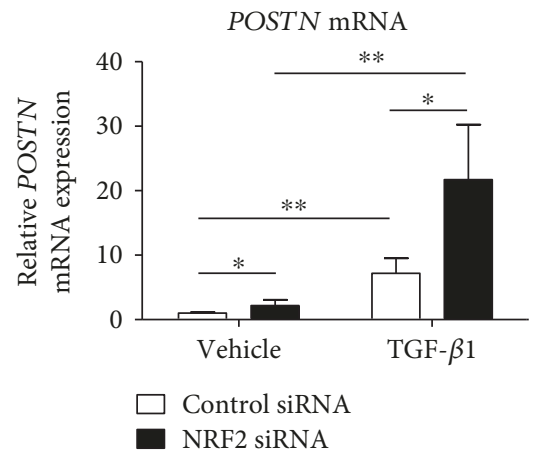

(b)

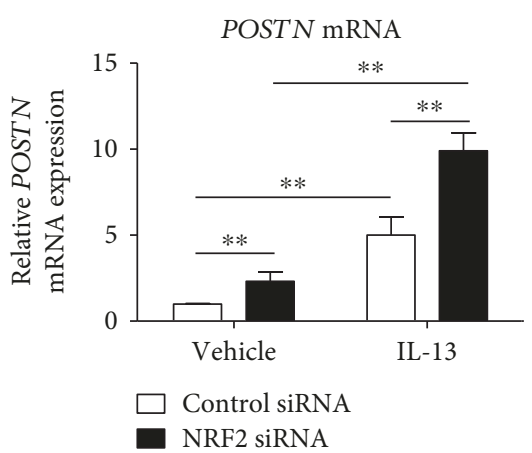

(c)

FIGURE 3: Knockdown of NRF2 upregulates the expression of periostin in dermal fibroblasts. (a) NHDF cells were transiently transfected with control siRNA or NRF2 siRNA for $24 \mathrm{~h}$, after which whole cell lysates or total RNA were extracted. The expression levels of NRF2 were normalized to that of GAPDH. NRF2 protein and mRNA expressions are depicted. (b, c) NHDF cells were transiently transfected with control siRNA or $5 \mathrm{nM} \mathrm{NRF2}$ siRNA for $24 \mathrm{~h}$ and then stimulated with $5 \mathrm{ng} / \mathrm{mL}$ TGF- $\beta 1$ (b) or IL- 13 (c). POSTN mRNA expression is depicted. The values were adjusted by GAPDH expression. The same experiments were performed three times. ${ }^{*} P<0.05,{ }^{* *} P<0.01$.

is associated with the induction of POSTN expression in dermal fibroblasts. First, we investigated whether TGF- $\beta 1$ and IL-13 induce the generation of intracellular ROS. Compared with unstimulated cells, both TGF- $\beta 1$ and IL-13 clearly induced intracellular ROS formation in NHDF cells (Figure 2(a)). As shown in Figures 2(b)-2(e), both TGF- $\beta 1$ and IL-13 upregulated the gene and protein expression of POSTN in dermal fibroblasts. At a noncytotoxic concentration, CIN inhibited the TGF- $\beta 1$-induced upregulation of the gene (Figure 2(b)) and protein (Figure 2(c)) expression of POSTN. It also inhibited the IL-13-induced upregulation of POSTN expression at the mRNA and protein levels (Figures 2(d) and 2(e)).

3.3. The Involvement of NRF2 in the Regulation of the $m R N A$ Expression of Periostin Induced by TGF- $\beta 1$ and IL-13. To elucidate the role of NRF2 in baseline and cytokine-induced expression of POSTN, we next knocked down NRF2 using NRF2 siRNA. The knockdown efficiency was $86.1 \pm 1.2 \%$ as assessed by qRT-PCR and was confirmed by Western blotting analysis (Figure 3(a)). Interestingly, the baseline expression of POSTN was significantly enhanced in the fibroblasts with NRF2 knockdown compared with those transfected with control siRNA (Figures 3(b) and 3(c)). In addition, both TGF- $\beta 1$ - and IL-13-mediated POSTN were further augmented in the fibroblasts with NRF2 knockdown compared with the levels in control fibroblasts (Figures 3(b) and 3(c)). Taken together, these results demonstrate that NRF2 downregulates both constitutive and inducible POSTN expression.

\subsection{NRF2 Knockdown Abolishes CIN-Mediated} Downregulation of POSTN. We next examined whether CIN-mediated downregulation of POSTN is dependent on NRF2. As shown in Figures 4(a) and 4(b), TGF- $\beta 1$ upregulated POSTN expression at the mRNA and protein levels, which CIN in turn inhibited. The capacity of CIN to inhibit TGF- $\beta 1$ signaling was abrogated in fibroblasts with NRF2 knockdown. Moreover, NRF2 knockdown abolished the inhibitory action of CIN on IL-13-mediated POSTN upregulation (Figures 4(c) and 4(d)). We next attempted to confirm that CIN actually inhibits TGF- $\beta 1$ - and IL-13-mediated oxidative stress. As expected, CIN inhibited both TGF- $\beta 1$ - and IL-13-induced production of ROS (Figure 5). In addition, we investigated the effects of CIN on TGF- $\beta 1$ - and IL-13induced gene expression of other profibrotic mediators, namely, tenascin-C (TNC), vascular endothelial growth factor (VEGF), and connective tissue growth factor (CTGF). As shown in Figure 6, the expression of all TNC, VEGF, and CTGF was upregulated by TGF- $\beta 1$ and IL-13, respectively, which was significantly inhibited by CIN in all cases. Notably, the CIN-mediated inhibitory capacity was again abolished in fibroblasts with NRF2 knockdown, suggesting that NRF2 signaling has multiple antifibrotic effects.

\section{Discussion}

Fibrosis is a complex condition mediated by profibrotic factors, an imbalance of collagen synthesis and degradation, upregulation of the extracellular matrix including POSTN and TNC, and increased oxidative stress. However, the mechanisms by which POSTN expression is regulated in fibrotic disease and their relationships with oxidative stress have remained elusive. In this study, we made the following key findings, which shed light on the mechanisms involved: (i) CIN activates NRF2 signaling and upregulates HMOX1 production in dermal fibroblasts. (ii) CIN inhibits POSTN expression induced by TGF- $\beta 1$ and IL-13 in dermal fibroblasts. (iii) Knockdown of NRF2 upregulates mRNA expression of constitutive and TGF- $\beta 1$ - and IL-13-induced POSTN. (iv) The CIN-mediated inhibition of POSTN expression induced by TGF- $\beta 1$ and IL-13 is dependent on NRF2 signaling. (v) The same kind of regulation via NRF2 signaling controls other profibrotic molecules, namely, TNC, VEGF, and CTGF. These findings suggest that CIN has a potential for treating fibrosis (Figure 7). 


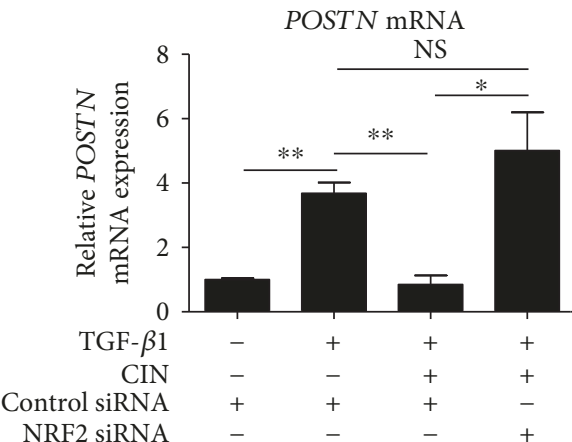

(a)

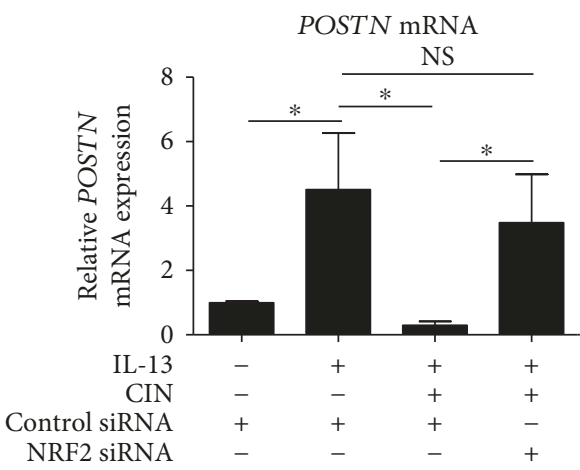

(c)

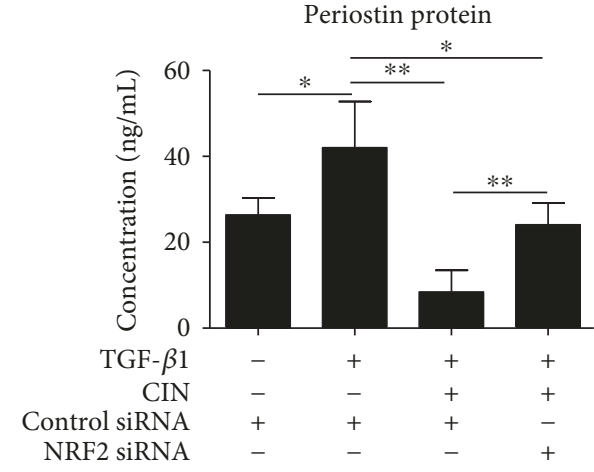

(b)

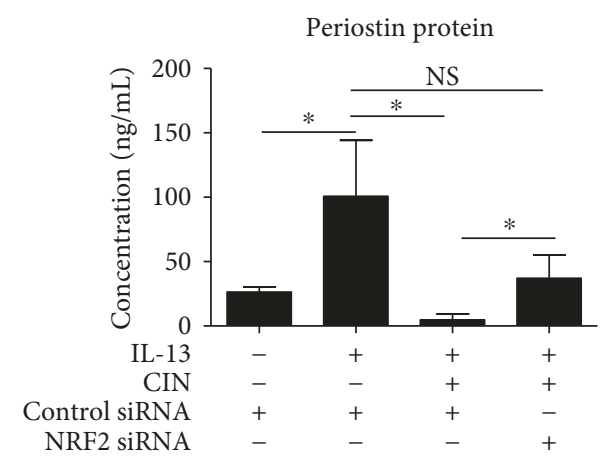

(d)

FIGURE 4: The involvement of NRF2 signaling in the downregulation of IL-13- and TGF- $\beta 1$-induced periostin expression by cinnamaldehyde. (a,b) Knockdown of NRF2 reverses the effects of cinnamaldehyde (CIN) on TGF- $\beta 1$-induced expression of periostin (POSTN) mRNA and protein. (c, d) Knockdown of NRF2 reverses the effects of CIN on IL-13-induced expression of POSTN mRNA and protein. NHDF cells were transiently transfected with control siRNA or NRF2 siRNA for $24 \mathrm{~h}$; next, cells were precultured in $25 \mu \mathrm{M}$ CIN for 30 min and then treated with $5 \mathrm{ng} / \mathrm{mL}$ TGF- $\beta 1$ or $50 \mathrm{ng} / \mathrm{mL}$ IL-13 or left untreated for $48 \mathrm{~h}$. Expression levels of POSTN mRNA and protein in the cell supernatants are depicted. The values were adjusted by GAPDH expression. The same experiments were performed three times. ${ }^{*} P<0.05,{ }^{* *} P<0.01$. NS: not significant.
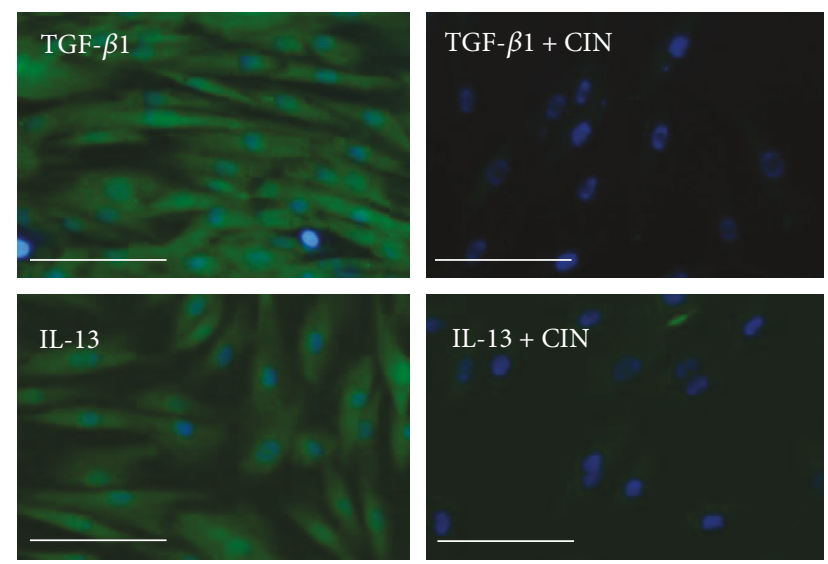

FIGURE 5: Effects of cinnamaldehyde on TGF- $\beta 1$ - and IL-13induced ROS production in dermal fibroblasts. NHDF cells were precultured in $25 \mu \mathrm{M}$ cinnamaldehyde (CIN) for $16 \mathrm{~h}$ and then treated with $20 \mathrm{ng} / \mathrm{mL}$ TGF- $\beta 1$ or $50 \mathrm{ng} / \mathrm{mL}$ IL- 13 for $20 \mathrm{~min}$. Cells were incubated with carboxy- $\mathrm{H}_{2}$ DCFDA for $25 \mathrm{~min}$, and ROS production was visualized by fluorescence microscopy. Scale bars, $100 \mu \mathrm{m}$.
Systemic fibrosing or sclerotic disorders such as scleroderma, pulmonary fibrosis, hepatic fibrosis, renal fibrosis, and cardiac fibrosis are life-threatening, but only very limited treatment modalities are available for them [39-42]. Although new therapies such as nintedanib, pirfenidone, tocilizumab, and rapamycin are emerging [13, 18, 40, 43, 44], additional safe and tolerable medicaments are demanded.

The profibrotic process in each organ involves unopposed collagen accumulation [11,45]. In addition to this collagen accumulation, most fibrotic lesions in systemic fibrotic diseases are associated with the upregulated expression of POSTN, which is mainly produced from activated fibroblasts [4, 6, 7, 21, 35, 46-49]. Notably, accumulated evidence indicates that stromal POSTN plays a key role in augmenting the production of collagen $[48,50]$ and suggests that POSTN is a potential target for the treatment of systemic fibrosis $[4,6,7,46]$.

Two different cytokines, TGF- $\beta 1$ and IL-13, upregulate POSTN expression via distinct signaling pathways $[1,2,9,33,34]$. The Kampo herbal medicine Keishibukuryo-gan inhibits collagen production in cultured fibroblasts derived from scleroderma patients [31]. Moreover, Japanese case series with 21 patients with systemic sclerosis 


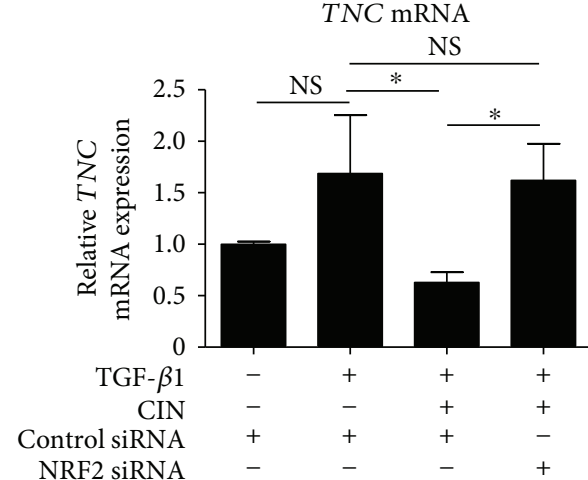

(a)

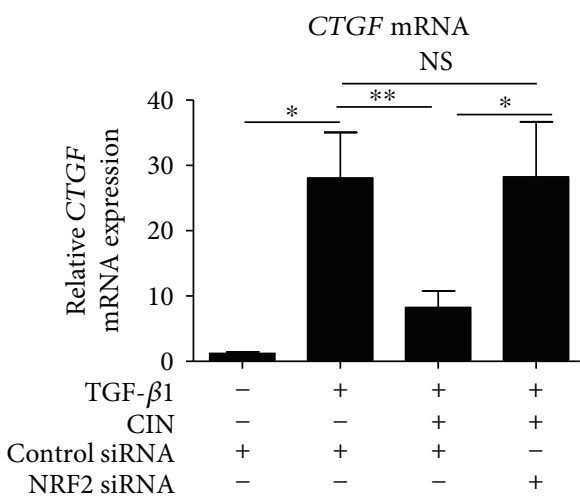

(c)

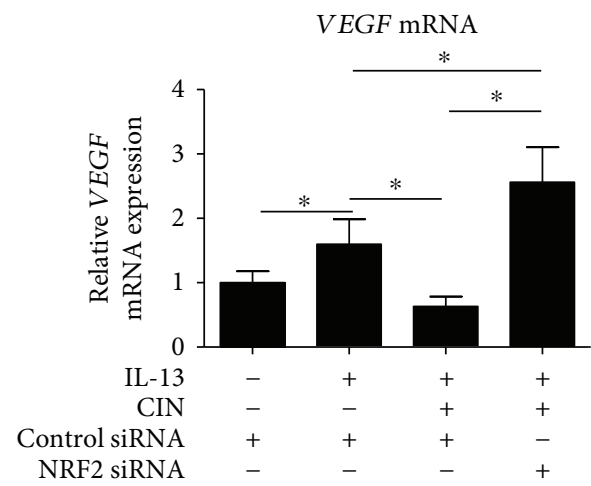

(e)

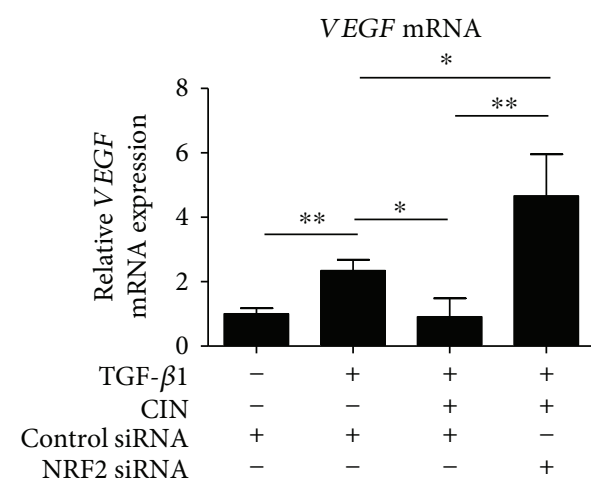

(b)

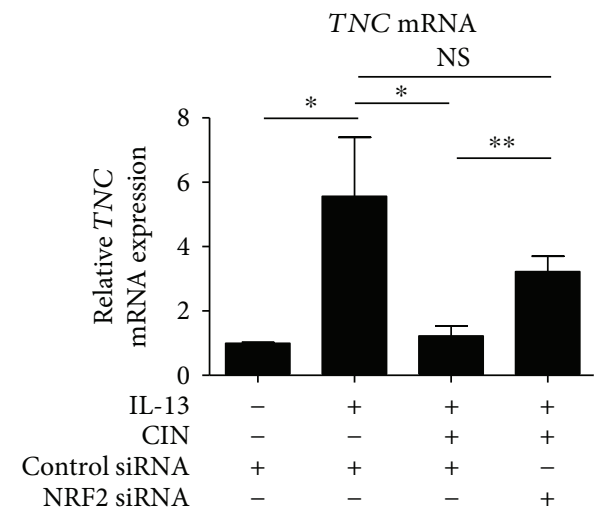

(d)

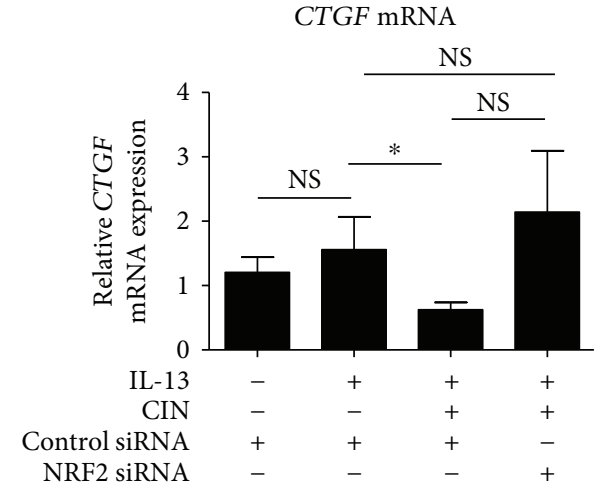

(f)

FIGURE 6: The involvement of NRF2 signaling in the downregulation of IL-13- and TGF- $\beta 1$-induced tenascin-C, VEGF, and CTGF mRNA expression by cinnamaldehyde. (a-c) Knockdown of NRF2 reverses the effects of cinnamaldehyde (CIN) on TGF- $\beta 1$-induced mRNA expression of TNC, VEGF, and CTGF. (d-f) Knockdown of NRF2 reverses the effects of CIN on IL-13-induced mRNA expression of TNC, $V E G F$, and CTGF. NHDF cells were transiently transfected with control siRNA or $5 \mathrm{nM} \mathrm{NRF2} \mathrm{siRNA} \mathrm{for} 24 \mathrm{~h}$; next, cells were precultured with $25 \mu \mathrm{M}$ CIN for $30 \mathrm{~min}$ and then treated with $5 \mathrm{ng} / \mathrm{mL}$ TGF- $\beta 1$ or $50 \mathrm{ng} / \mathrm{mL}$ IL-13 or left untreated for $48 \mathrm{~h}$. mRNA expression levels of TNC, VEGF, and CTGF are depicted. The values were adjusted by GAPDH expression. The same experiments were performed three times. ${ }^{*} P<0.05,{ }^{* *} P<0.01$. NS: not significant.

showed that the Kampo herbal medicine including Keishibukuryo-gan improves clinical sclerotic symptoms in 19 scleroderma patients (90.4\%) [32]. Based on this background, we speculated that CIN, a major active ingredient of Keishi-bukuryo-gan, might downregulate POSTN expression in fibroblasts.

In this study, we indeed demonstrated that CIN inhibited POSTN expression in fibroblasts stimulated by either TGF- $\beta 1$ or IL-13 signaling. CIN is a safe natural product derived from cinnamon, which is a commonly used spice. CIN is also known to be a potent antioxidant phytochemical that activates the antioxidant NRF2/HMOX1 and NQO1 axis $[29,51]$. As TGF- $\beta 1$ and IL-13 signaling induces oxidative stress by producing ROS [27, 52], we hypothesized that CIN/NRF2-mediated antioxidant activity contributes to the inhibitory action on both TGF- $\beta 1$-induced and IL-13- 


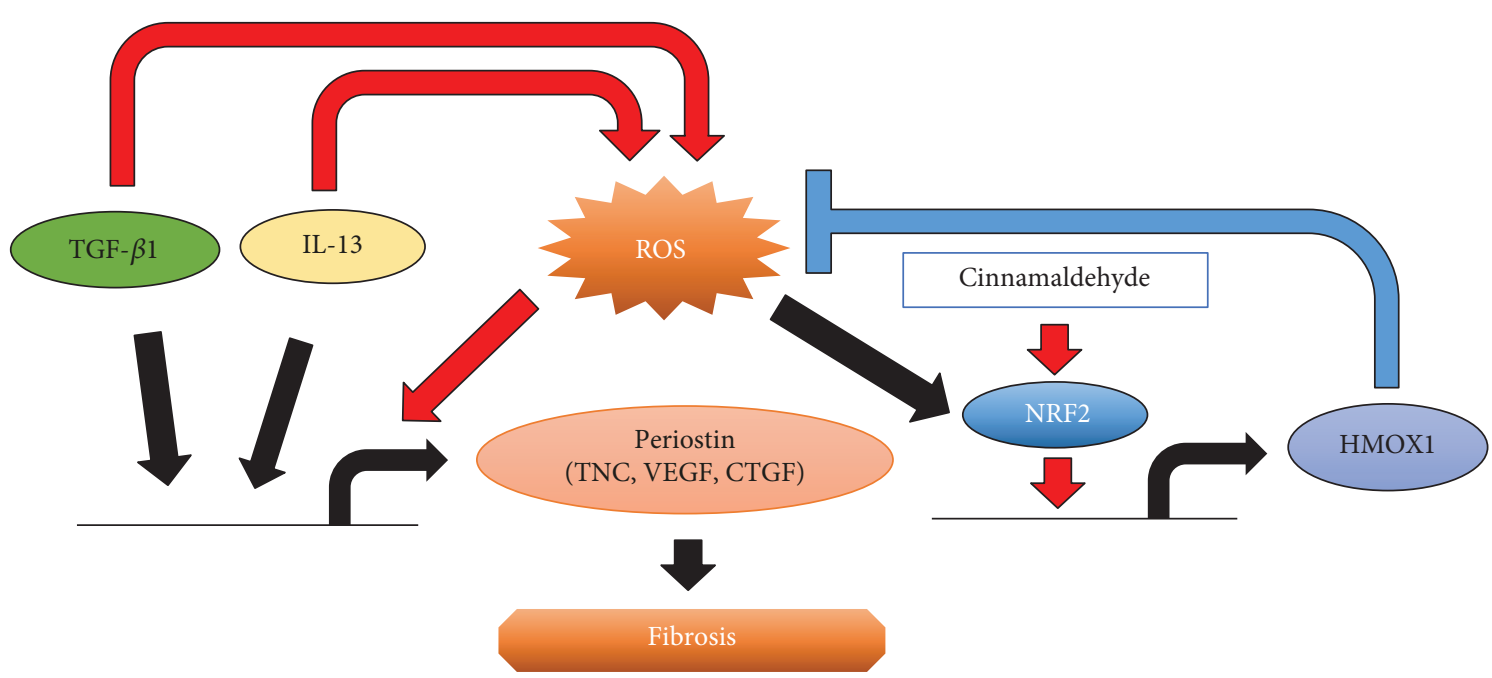

FIGURE 7: Schematic model of the molecular mechanism by which cinnamaldehyde inhibits both IL-13- and TGF- $\beta 1$-mediated periostin expression in fibroblasts. Cinnamaldehyde induces NRF2 nuclear translocation and HMOX1 production, which downregulates IL-13- and TGF- $\beta 1$-mediated POSTN, TNC, VEGF, and CTGF expression by reducing ROS production, followed by the attenuation of fibrosis.

induced POSTN upregulation. As expected, under our experimental conditions, CIN induced the cytoplasmic-tonuclear translocation of NRF2 and the NRF2 activation upregulated the expression of HMOX1 and NQO1 at the mRNA level, but only the expression of HMOX1 at the protein level (Figure 1). Various compounds including CIN were shown to promote NRF2-mediated HMOX1 and NQO1 genes in several cells such as fibroblasts, keratinocytes, endothelial cells, epithelial colon cells, and cardiomyocytes $[29,51,53-55]$. Our present findings and previous study demonstrate that activation of cellular antioxidant response orchestrated by NRF2 has been revealed through primarily increased HMOX1 in human dermal fibroblasts [54]. The baseline as well as TGF- $\beta 1$-induced and IL-13induced POSTN upregulation was augmented in fibroblasts with NRF2 knockdown. Moreover, the CIN-induced inhibition of POSTN upregulation was abrogated by NRF2 knockdown upon both TGF- $\beta 1$ and IL-13 stimulation. As a profibrotic cytokine, IL-13 participates in the fibrosis of skin and internal organs such as the liver and is considered to play an important role in inflammatory and fibrotic processes $[56,57]$. Moreover, fibrosis is one of the key pathological features of airway remodeling in asthma, and the current study demonstrated that activating the NRF2 signaling pathway ameliorates ovalbumin-induced allergic airway inflammation, which is also supported by our present findings [58]. This study showed that both TGF- $\beta 1$ and IL- 13 stimulation induced the intracellular ROS production and the activated NRF2 signaling pathway suppressed both cytokine signaling in dermal fibroblasts. Although the underlying mechanisms are yet to be clarified, the ROS production by both TGF- $\beta 1$ and IL-13 stimulation exacerbates fibrosis through upregulation of profibrotic molecules in synergism. These results suggest that the effect of TGF- $\beta 1$ and IL-13 stimulation may be linked with the requirement of ROS production, and each signaling pathway was regulated by the NRF2/HMOX1 pathway. Furthermore, oxidative stress appeared to coordinately upregulate the expression of other profibrotic molecules such as VEGF, CTGF, and TNC. To the best of our knowledge, this is the first report demonstrating that the CIN/NRF2 pathway regulates POSTN expression induced by TGF- $\beta 1$ and IL- 13 .

In conclusion, the results obtained here suggest that CIN is a broad inhibitor of POSTN expression covering both TGF- $\beta 1$ and IL-13 signaling. Herbs and medicaments containing high level of CIN, such as Keishi-bukuryo-gan, may thus be applicable for the treatment and prevention of systemic fibrotic diseases.

\section{Data Availability}

No data were used to support this study.

\section{Conflicts of Interest}

The authors have no actual or potential conflicts of interest associated with this work.

\section{Acknowledgments}

This work was partly supported by a grant from the Japanese Ministry of Health, Labour, and Welfare (Research on Food Safety; H27-Designated Research-017).

\section{References}

[1] K. Izuhara, S. Nunomura, Y. Nanri et al., "Periostin in inflammation and allergy," Cellular and Molecular Life Sciences, vol. 74, no. 23, pp. 4293-4303, 2017.

[2] H. Murota, Y. Lingli, and I. Katayama, "Periostin in the pathogenesis of skin diseases," Cellular and Molecular Life Sciences, vol. 74, no. 23, pp. 4321-4328, 2017.

[3] M. Masuoka, H. Shiraishi, S. Ohta et al., "Periostin promotes chronic allergic inflammation in response to Th2 cytokines," 
The Journal of Clinical Investigation, vol. 122, no. 7, pp. 25902600, 2012.

[4] L. Yang, S. Serada, M. Fujimoto et al., "Periostin facilitates skin sclerosis via PI3K/Akt dependent mechanism in a mouse model of scleroderma," PLoS One, vol. 7, no. 7, article e41994, 2012.

[5] Y. Kotobuki, L. Yang, S. Serada et al., "Periostin accelerates human malignant melanoma progression by modifying the melanoma microenvironment," Pigment Cell \& Melanoma Research, vol. 27, no. 4, pp. 630-639, 2014.

[6] M. W. Kim, J. T. Park, J. H. Kim et al., "Periostin in mature stage localized scleroderma," Annals of Dermatology, vol. 29, no. 3, pp. 268-275, 2017.

[7] Y. Yamaguchi, J. Ono, M. Masuoka et al., "Serum periostin levels are correlated with progressive skin sclerosis in patients with systemic sclerosis," The British Journal of Dermatology, vol. 168, no. 4, pp. 717-725, 2013.

[8] S. Ohta, M. Okamoto, K. Fujimoto et al., "The usefulness of monomeric periostin as a biomarker for idiopathic pulmonary fibrosis," PLoS One, vol. 12, no. 3, article e0174547, 2017.

[9] L. Hong, D. Shejiao, C. Fenrong, Z. Gang, and D. Lei, "Periostin down-regulation attenuates the pro-fibrogenic response of hepatic stellate cells induced by TGF- $\beta 1$," Journal of Cellular and Molecular Medicine, vol. 19, no. 10, pp. 2462-2468, 2015.

[10] K. M. Langberg, J. M. Kapo, and T. H. Taddei, "Palliative care in decompensated cirrhosis: a review," Liver International, vol. 38, no. 5, pp. 768-775, 2018.

[11] Y. Asano, "Recent advances in animal models of systemic sclerosis," The Journal of Dermatology, vol. 43, no. 1, pp. 19-28, 2016.

[12] Y. Asano, "Systemic sclerosis," The Journal of Dermatology, vol. 45, no. 2, pp. 128-138, 2018.

[13] M. Furue, C. Mitoma, H. Mitoma et al., "Pathogenesis of systemic sclerosis-current concept and emerging treatments," Immunologic Research, vol. 65, no. 4, pp. 790-797, 2017.

[14] S. Numata, Y. Iwata, M. Kobayashi, T. Sato, and K. Sugiura, "Successful treatment of continuous intra-arterial administration of prostaglandin E1, urokinase and heparin for intractable digital ulcers by upper extremity arterial occlusion in diffuse cutaneous systemic sclerosis patient," The Journal of Dermatology, vol. 44, no. 10, pp. e254-e255, 2017.

[15] Y. Hamaguchi, T. Sumida, Y. Kawaguchi et al., "Safety and tolerability of bosentan for digital ulcers in Japanese patients with systemic sclerosis: prospective, multicenter, open-label study," The Journal of Dermatology, vol. 44, no. 1, pp. 13-17, 2017.

[16] Y. Matsuura-otsuki, T. Hanafusa, T. Namiki et al., "Peripheral arterial bypass surgery for intractable wounds caused by limited cutaneous systemic sclerosis," The Journal of Dermatology, vol. 43, no. 12, pp. 1447-1448, 2016.

[17] S.-I. Motegi, K. Yamada, S. Toki et al., "Beneficial effect of botulinum toxin A on Raynaud's phenomenon in Japanese patients with systemic sclerosis: a prospective, case series study," The Journal of Dermatology, vol. 43, no. 1, pp. 56-62, 2016.

[18] K. Yanaba, "Strategy for treatment of fibrosis in systemic sclerosis: present and future," The Journal of Dermatology, vol. 43, no. 1, pp. 46-55, 2016.

[19] G. Latella, "Concise commentary: is Nrf2 a master regulator of intestinal fibrosis?," Digestive Diseases and Sciences, vol. 63, no. 2, pp. 381-382, 2018.
[20] G. Traver, S. Mont, D. Gius et al., "Loss of Nrf2 promotes alveolar type 2 cell loss in irradiated, fibrotic lung," Free Radical Biology \& Medicine, vol. 112, pp. 578-586, 2017.

[21] W. Sun, X. Liu, H. Zhang et al., "Epigallocatechin gallate upregulates NRF2 to prevent diabetic nephropathy via disabling KEAP1," Free Radical Biology \& Medicine, vol. 108, pp. 840857, 2017.

[22] J. Wei, H. Zhu, G. Lord et al., "Nrf2 exerts cell-autonomous antifibrotic effects: compromised function in systemic sclerosis and therapeutic rescue with a novel heterocyclic chalcone derivative," Translational Research, vol. 183, pp. 71-86.e1, 2017.

[23] N. Kikuchi, Y. Ishii, Y. Morishima et al., "Nrf2 protects against pulmonary fibrosis by regulating the lung oxidant level and Th1/Th2 balance," Respiratory Research, vol. 11, no. 1, p. 31, 2010.

[24] K. Shen, X. Feng, H. Pan, F. Zhang, H. Xie, and S. Zheng, "Baicalin ameliorates experimental liver cholestasis in mice by modulation of oxidative stress, inflammation, and NRF2 transcription factor," Oxidative Medicine and Cellular Longevity, vol. 2017, Article ID 6169128, 11 pages, 2017.

[25] A. S. Arellano-Buendía, M. Tostado-González, F. E. GarcíaArroyo et al., "Anti-inflammatory therapy modulates Nrf2Keap1 in kidney from rats with diabetes," Oxidative Medicine and Cellular Longevity, vol. 2016, Article ID 4693801, 11 pages, 2016.

[26] C. Zeng, P. Zhong, Y. Zhao et al., "Curcumin protects hearts from FFA-induced injury by activating Nrf2 and inactivating NF- $\kappa$ B both in vitro and in vivo," Journal of Molecular and Cellular Cardiology, vol. 79, pp. 1-12, 2015.

[27] Y. Guan, Y. Tan, W. Liu et al., "NF-E2-related factor 2 suppresses intestinal fibrosis by inhibiting reactive oxygen species-dependent TGF- $\beta 1 /$ SMADs pathway," Digestive Diseases and Sciences, vol. 63, no. 2, pp. 366-380, 2018.

[28] M. Furue, H. Uchi, C. Mitoma et al., "Antioxidants for healthy skin: the emerging role of aryl hydrocarbon receptors and nuclear factor-erythroid 2-related factor-2," Nutrients, vol. 9, no. 3, p. 223, 2017.

[29] H. Uchi, M. Yasumatsu, S. Morino-Koga, C. Mitoma, and M. Furue, "Inhibition of aryl hydrocarbon receptor signaling and induction of NRF2-mediated antioxidant activity by cinnamaldehyde in human keratinocytes," Journal of Dermatological Science, vol. 85, no. 1, pp. 36-43, 2017.

[30] N. Y. Kim, S. G. Ahn, and S. A. Kim, "Cinnamaldehyde protects human dental pulp cells against oxidative stress through the Nrf2/HO-1-dependent antioxidant response," European Journal of Pharmacology, vol. 815, pp. 73-79, 2017.

[31] F. Y. Sheng, A. Ohta, and M. Yamaguchi, "Inhibition of collagen production by traditional Chinese herbal medicine in scleroderma fibroblast cultures," Internal Medicine, vol. 33, no. 8, pp. 466-471, 1994.

[32] J. Dokura, E. Tahara, S. Ota et al., "Clinical efficacy of Kampo treatments for systemic sclerosis," Kampo No Rinsho, vol. 60, no. 5, pp. 795-806, 2013.

[33] K. Horiuchi, N. Amizuka, S. Takeshita et al., "Identification and characterization of a novel protein, periostin, with restricted expression to periosteum and periodontal ligament and increased expression by transforming growth factor beta," Journal of Bone and Mineral Research, vol. 14, no. 7, pp. 12391249, 1999. 
[34] G. Takayama, K. Arima, T. Kanaji et al., "Periostin: a novel component of subepithelial fibrosis of bronchial asthma downstream of IL-4 and IL-13 signals," The Journal of Allergy and Clinical Immunology, vol. 118, no. 1, pp. 98-104, 2006.

[35] M. Okamoto, T. Hoshino, Y. Kitasato et al., "Periostin, a matrix protein, is a novel biomarker for idiopathic interstitial pneumonias," The European Respiratory Journal, vol. 37, no. 5, pp. 1119-1127, 2011.

[36] M. Murai, G. Tsuji, A. Hashimoto-Hachiya, Y. Kawakami, M. Furue, and C. Mitoma, "An endogenous tryptophan photo-product, FICZ, is potentially involved in photo-aging by reducing TGF- $\beta$-regulated collagen homeostasis," Journal of Dermatological Science, vol. 89, no. 1, pp. 19-26, 2018.

[37] H. Wu, L. Chen, J. Xie et al., "Periostin expression induced by oxidative stress contributes to myocardial fibrosis in a rat model of high salt-induced hypertension," Molecular Medicine Reports, vol. 14, no. 1, pp. 776-782, 2016.

[38] Z. Zhong and Y. Tang, "Upregulation of periostin prevents high glucose-induced mitochondrial apoptosis in human umbilical vein endothelial cells via activation of $\mathrm{Nrf} 2 / \mathrm{HO}-1$ signaling," Cellular Physiology and Biochemistry, vol. 39, no. 1, pp. 71-80, 2016.

[39] C. P. Denton, M. Hughes, N. Gak et al., "BSR and BHPR guideline for the treatment of systemic sclerosis," Rheumatology, vol. 55, no. 10, pp. 1906-1910, 2016.

[40] J. Behr, A. Günther, F. Bonella et al., "German guideline for idiopathic pulmonary fibrosis - update on pharmacological therapies 2017," Pneumologie, vol. 72, no. 2, pp. 155-168, 2018.

[41] P. Harrison, B. J. Hogan, L. Floros, and E. Davies, “Assessment and management of cirrhosis in people older than 16 years: summary of NICE guidance," BMJ, vol. 354, article i2850, 2016.

[42] S.-Y. Lee, S. I. Kim, and M. E. Choi, "Therapeutic targets for treating fibrotic kidney diseases," Translational Research, vol. 165, no. 4, pp. 512-530, 2015.

[43] T. Taniguchi, Y. Asano, T. Fukasawa, A. Yoshizaki, and S. Sato, "Critical contribution of the interleukin-6/signal transducer and activator of transcription 3 axis to vasculopathy associated with systemic sclerosis," The Journal of Dermatology, vol. 44, no. 8, pp. 967-971, 2017.

[44] A. Yoshizaki, "B lymphocytes in systemic sclerosis: abnormalities and therapeutic targets," The Journal of Dermatology, vol. 43, no. 1, pp. 39-45, 2016.

[45] M. Hasegawa, "Biomarkers in systemic sclerosis: their potential to predict clinical courses," The Journal of Dermatology, vol. 43 , no. 1 , pp. 29-38, 2016.

[46] J. H. Hwang, S. H. Yang, Y. C. Kim et al., "Experimental inhibition of periostin attenuates kidney fibrosis," American Journal of Nephrology, vol. 46, no. 6, pp. 501-517, 2017.

[47] N. Prakoura and C. Chatziantoniou, "Periostin in kidney diseases," Cellular and Molecular Life Sciences, vol. 74, no. 23, pp. 4315-4320, 2017.

[48] K. Takeda, R. Noguchi, M. Kitade et al., "Periostin cross-reacts with the renin-angiotensin system during liver fibrosis development," Molecular Medicine Reports, vol. 16, no. 5, pp. 5752-5758, 2017.

[49] N. M. Landry, S. Cohen, and I. M. C. Dixon, "Periostin in cardiovascular disease and development: a tale of two distinct roles," Basic Research in Cardiology, vol. 113, no. 1, p. 1, 2018.
[50] D. N. O’Dwyer and B. B. Moore, “The role of periostin in lung fibrosis and airway remodeling," Cellular and Molecular Life Sciences, vol. 74, no. 23, pp. 4305-4314, 2017.

[51] F. Wang, C. Pu, P. Zhou et al., "Cinnamaldehyde prevents endothelial dysfunction induced by high glucose by activating Nrf2," Cellular Physiology and Biochemistry, vol. 36, no. 1, pp. 315-324, 2015.

[52] S. Y. Won, S. R. Kim, S. Maeng, and B. K. Jin, "Interleukin-13/ Interleukin-4-induced oxidative stress contributes to death of prothrombinkringle-2 (pKr-2)-activated microglia," Journal of Neuroimmunology, vol. 265, no. 1-2, pp. 36-42, 2013.

[53] G. T. Wondrak, C. M. Cabello, N. F. Villeneuve et al., "Cinnamoyl-based Nrf2-activators targeting human skin cell photooxidative stress," Free Radical Biology \& Medicine, vol. 45, no. 4, pp. 385-395, 2008.

[54] Y. C. Hseu, M. Korivi, F. Y. Lin et al., “Trans-cinnamic acid attenuates UVA-induced photoaging through inhibition of AP-1 activation and induction of Nrf2-mediated antioxidant genes in human skin fibroblasts," Journal of Dermatological Science, vol. 90, no. 2, pp. 123-134, 2018.

[55] X. Li, D. Wu, and Y. Tian, "Fibroblast growth factor 19 protects the heart from oxidative stress-induced diabetic cardiomyopathy via activation of AMPK/Nrf2/HO-1 pathway," Biochemical and Biophysical Research Communications, vol. 502, no. 1, pp. 62-68, 2018.

[56] X. L. Huang, Y. J. Wang, J. W. Yan et al., "Role of antiinflammatory cytokines IL-4 and IL-13 in systemic sclerosis," Inflammation Research, vol. 64, no. 3-4, pp. 151-159, 2015.

[57] K. M. Hart, T. Fabre, J. C. Sciurba et al., "Type 2 immunity is protective in metabolic disease but exacerbates NAFLD collaboratively with TGF- $\beta$," Science Translational Medicine, vol. 9, no. 396, article eaal3694, 2017.

[58] P. Ye, X. L. Yang, X. Chen, and C. Shi, "Hyperoside attenuates OVA-induced allergic airway inflammation by activating Nrf2," International Immunopharmacology, vol. 44, pp. 168$173,2017$. 


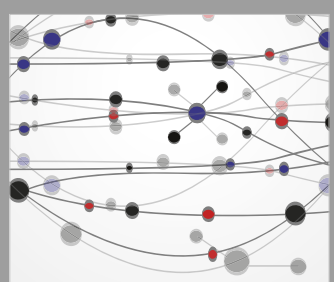

The Scientific World Journal
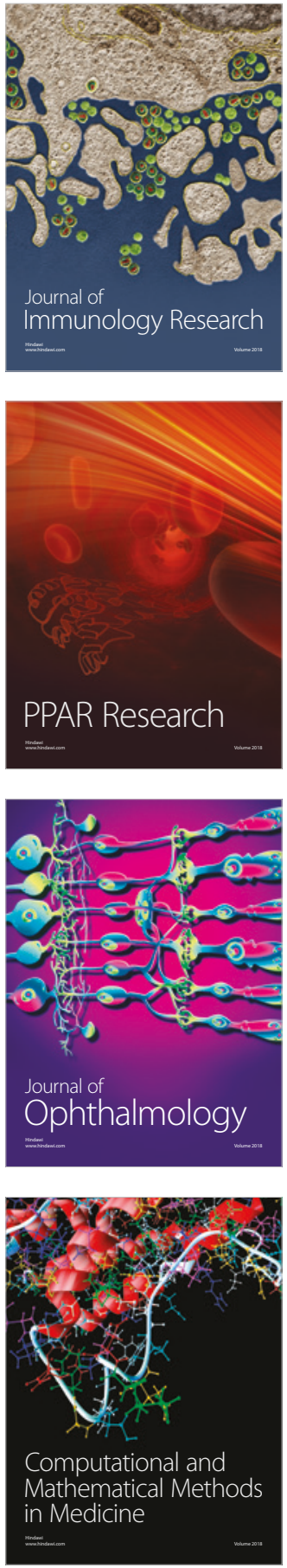

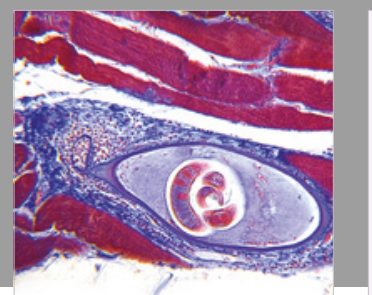

Gastroenterology Research and Practice

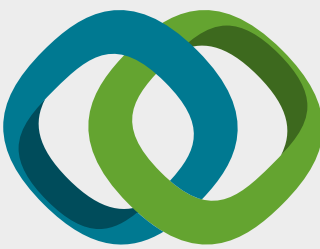

\section{Hindawi}

Submit your manuscripts at

www.hindawi.com
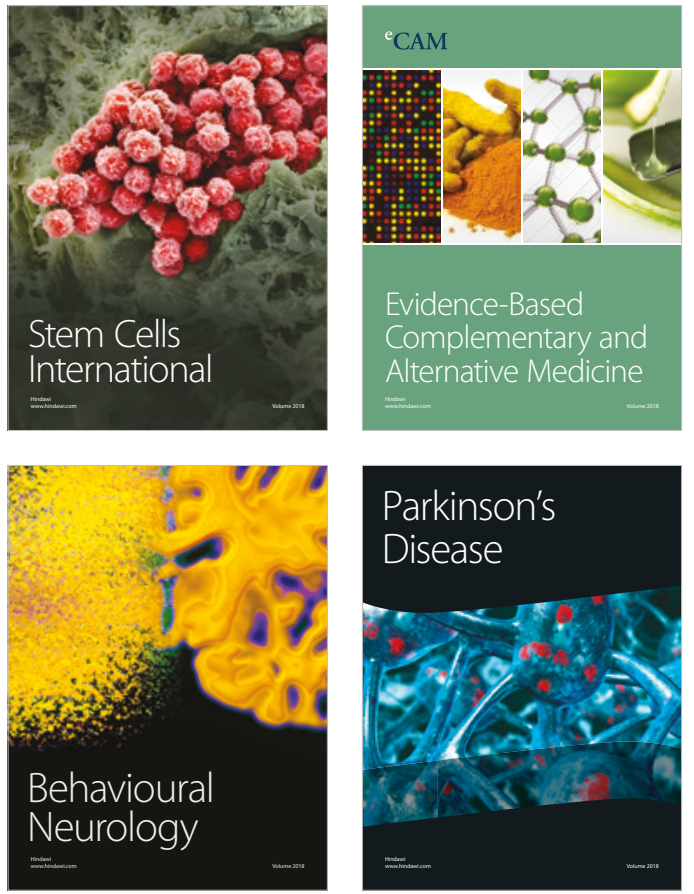

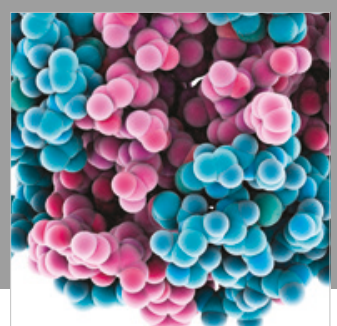

ournal of

Diabetes Research

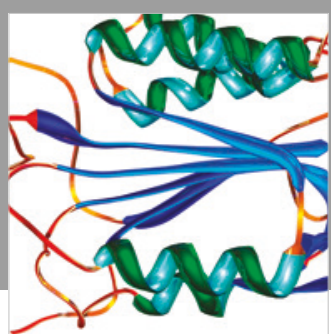

Disease Markers
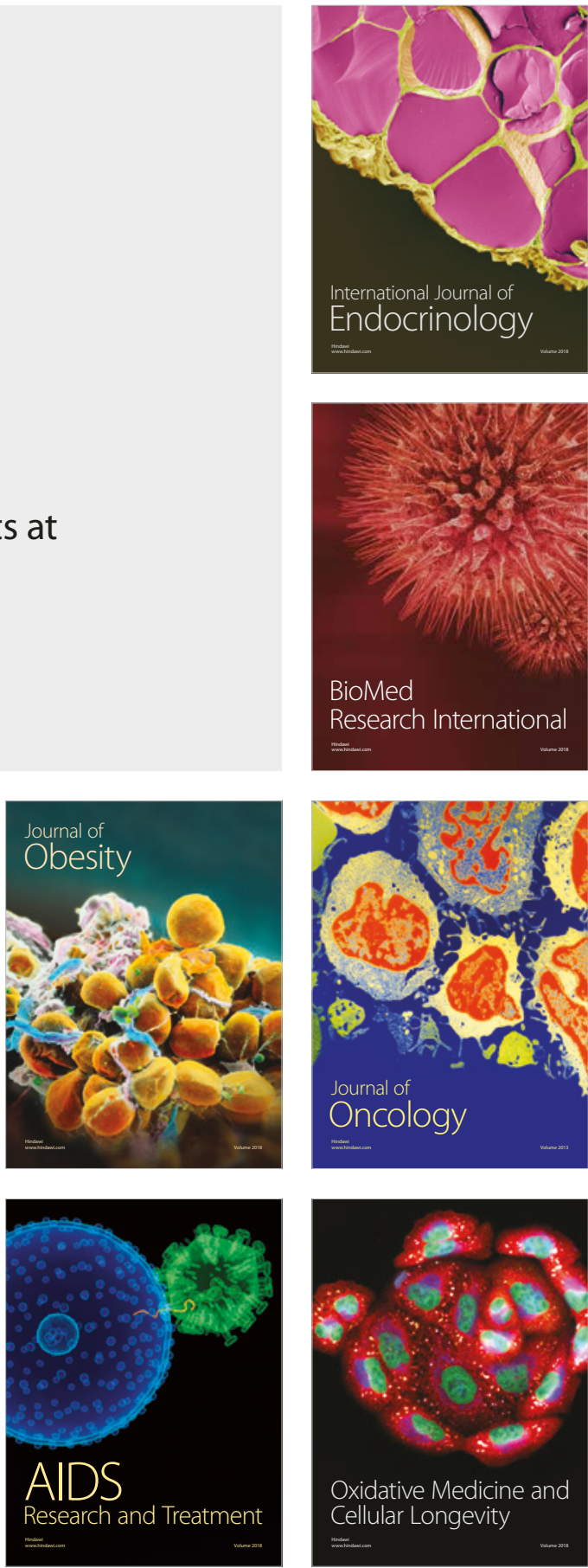